\title{
EVIDENCE FOR A LATITUDINAL GRADIENT OF THE COSMIC RAY INTENSITY ASSOCIATED WITH A CHANGE IN THE TILT OF THE HELIOSPHERIC CURRENT SHEET
}

\author{
S. P. Christon* and E. C. Stone \\ California Institute of Technology, Pasadena, CA 91125
}

J. T. Hoeksema

Stanford University, Stanford, CA 94305

\begin{abstract}
Since mid-1985, the average flux of $>70$ $\mathrm{MeV} /$ nucleon cosmic rays at Voyager $2\left(\mathrm{r}_{2} \sim 17 \mathrm{AU}\right.$, $\Theta_{2} \sim 0^{\circ}$ ) has been $\sim 3-5 \%$ greater than that at Voyager $1\left(\mathrm{r}_{1} \sim 24 \mathrm{AU}, \Theta_{1} \sim 26^{\circ} \mathrm{N}\right)$. This is the first direct observation over such a large radial range in which the galactic cosmic ray flux closer to the sun is higher than the flux farther from the sun for an extended period of time. This observation is consistent with the presence of a negative latitudinal gradient $\mathrm{G}_{\Theta}=-0.36 \pm 0.05$ (or $-0.60 \pm 0.08) \% / \mathrm{deg}$, assuming a coexistent radial gradient $G_{r}$ of 1 (or 2) \%/AU. We suggest that the appearance of this persistent negative latitudinal gradient may be due to the abrupt, large decrease of the heliospheric current sheet tilt to $\sim 20^{\circ}$ in early 1985 .
\end{abstract}

\section{Introduction}

In an earlier paper [Christon et al., 1986] we derived an average latitudinal component of the cosmic ray gradient with respect to the heliospheric current sheet of $\mathrm{G}_{\Lambda}=-0.22 \pm 0.03 \% / \mathrm{deg}$ during periods of restricted interplanetary conditions from 1981 to 1983. These results were in qualitative agreement with cosmic ray propagation models incorporating particle drifts. Since then, the general level of solar activity has decreased and the maximum latitudinal extent of the heliospheric current sheet has also decreased (e.g., Smith and Thomas [1986]), leading to the possibility that a negative latitudinal gradient might be observed by the Voyagers under less restrictive interplanetary conditions.

The first observational evidence for a persistent large-scale negative cosmic ray latitudinal gradient is presented in this letter and in a companion paper by McDonald and Lal [1986]. The radial and latitudinal separations of the observation points are $\sim 7 \mathrm{AU}$ and $\sim 26^{\circ}$, respectively, and the negative latitudinal gradient has persisted for over one-half year (7 solar rotations).

\section{Observations}

Our cosmic ray data are from the Voyager 1 (distance from the sun $r_{1} \sim 24 \mathrm{AU}$, heliographic latitude

*Present address: Johns Hopkins University Applied Physics Laboratory, Laurel, MD 20707

Copyright 1986 by the American Geophysical Union.

Paper number 6L6215.

0094-8276/86/006L-6215\$03.00
$\Theta_{1} \sim 26^{\circ} \mathrm{N}$ in mid-1985) and Voyager $2\left(\mathrm{r}_{2} \sim 17 \mathrm{AU}\right.$, $\Theta_{2} \sim 0^{\circ}$ in mid-1985) spacecraft. Concurrent 26-day solar rotation averages of the integral counting rates of $>70 \mathrm{MeV} /$ nucleon cosmic ray hydrogen and helium are determined for particles penetrating a stack of solid state detectors [Stone et al., 1977]. The median energy of the cosmic rays contributing to this counting rate is $\sim 1 \mathrm{GeV}$. In Figures $1 \mathrm{~A}$ and $1 \mathrm{~B}, 26$-day averages of normalized $\mathrm{H}$ and $\mathrm{He}$ counting rates at the Voyagers are plotted versus Voyager 2 observation time. The counting rates were normalized during a quiet period in 1977 when the spacecraft were at the same radial distance from the sun (e.g., Roelof et al. [1983]). The collection times of the Voyager 1 data were adjusted for $500 \mathrm{~km} / \mathrm{s}$ radial propagation from Voyager 2 to Voyager 1 before these 'shifted' 26-day Voyager 1 averages were accumulated. We use $500 \mathrm{~km} / \mathrm{s}$ for the radial propagation speed since this is the average apparent outward propagation speed of corotating interaction regions in 1983 and early 1984 [Christon and Stone, 1985a; Burlaga et al., 1985]. Shifting the data for the propagation delay should minimize the effects due to temporal variations convected with the solar wind and provide reasonable organization of data obtained at different heliocentric distances.

Latitudinal and radial gradients are calculated from the data using

$$
\ln \Gamma=\mathrm{G}_{\mathrm{r}} \Delta \mathrm{r}+\mathrm{G}_{\Theta} \Delta \Theta
$$

where $\Gamma$ is the ratio of cosmic ray flux measurements at Voyager 1 to those at Voyager $2, G_{r}\left(G_{\Theta}\right)$ is the radial (latitudinal) component of the cosmic ray gradient, and $\Delta \mathrm{r}(\Delta \Theta)$ is the radial (latitudinal) difference of the Voyagers (see Christon et al. [1986]). Since Voyager latitudinal and radial separations are both increasing steadily (see ephemeris at the top of Figure 1) and since effects due to both separations may be intermixed, we initially investigate temporal variations of the apparent radial gradient $G_{r}$, evaluated assuming $G_{\Theta}=0$ (see Figures $1 \mathrm{C}$ and $1 D)$. In 1984 the values of the apparent $G_{r}$ for hydrogen range from $\sim 0.2$ to $\sim 1.2 \% / \mathrm{AU}$, consistent with those evaluated by Venkatesan et al. [1985]. However, we note that these apparent values of $G_{r}$ from comparisons of Voyager 1 and Voyager 2 are less than half the values of $G_{r}$ from comparisons of Voyager 2 and Pioneer 10 which is also close to the heliographic equator but on the opposite side of the solar system [McDonald and Lal, 1986]. This suggests 


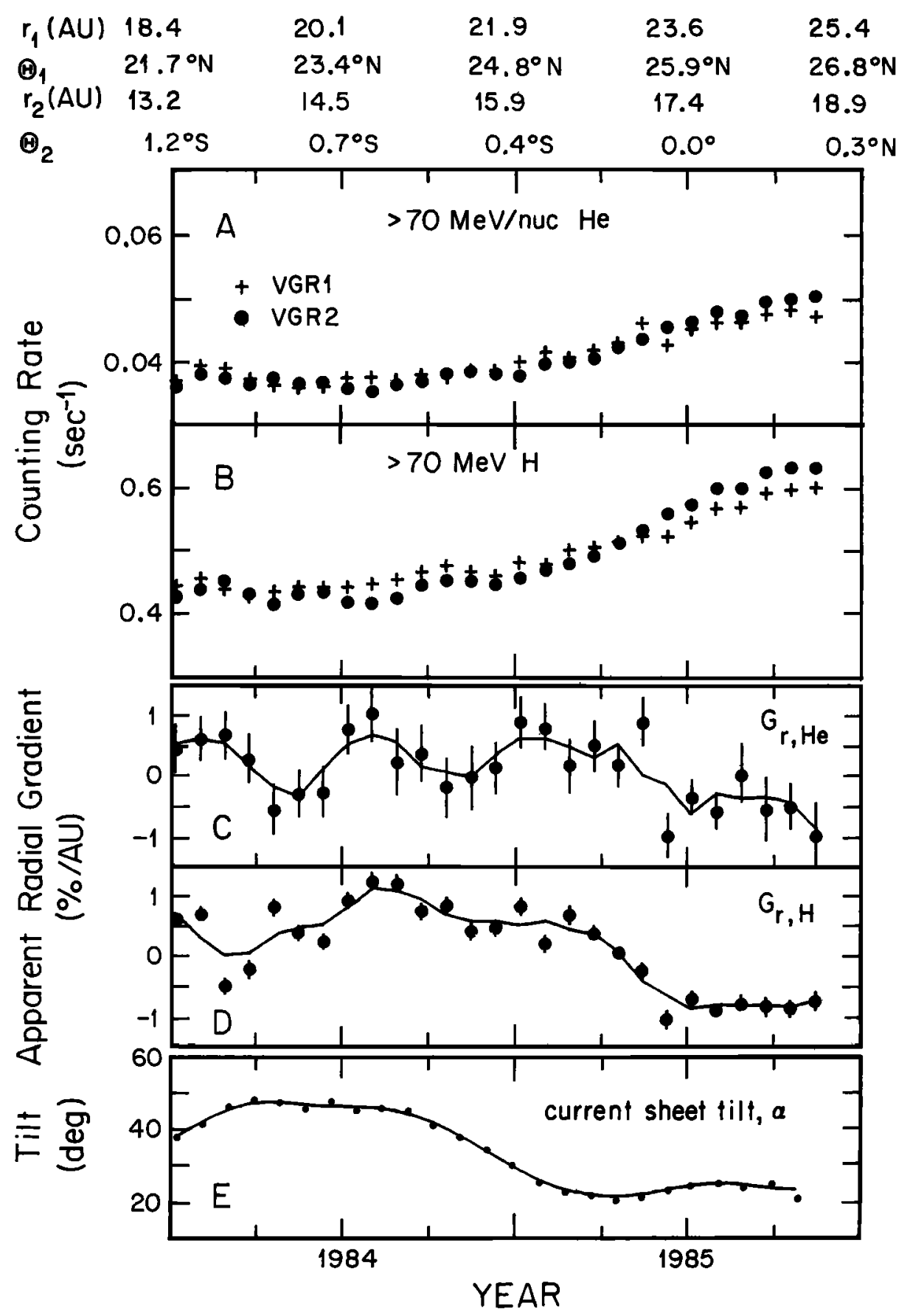

Fig. 1. Twenty-six day averages of $>70 \mathrm{MeV}$ cosmic ray $\mathrm{H}$ and He normalized counting rates (panels $\mathrm{A}$ and $\mathrm{B}$ ) and apparent radial gradients assuming a zero latitudinal gradient (panels $C$ and D) from Voyager 1 (plus) and Voyager 2 (diamond) are plotted. Note that a negative apparent radial gradient $G_{r}$, as observed following day 136, 1985, will result if there is a sufficiently large negative latitudinal gradient. Solar rotation averages of $\alpha$, the current sheet tilt, are plotted in panel E. Smooth curves are three-period running averages of the data.

that latitude effects are already measurable in 1984. Early in 1985, the apparent value of $G_{r}$ for both hydrogen and helium becomes negative and remains so, reflecting the presence of the higher fluxes at Voyager 2. We interpret these persistent negative values of the apparent $G_{r}$ to indicate that there is a persistent negative latitudinal gradient.

We evaluate $G_{\Theta}$ in 1985 using Equation 1 and typical values of 1 and $2 \% / A U$ for the actual $G_{r} ; 1 \% / A U$ agrees with our measurements in mid-1984 and is a reasonable lower limit, while $2 \% / \mathrm{AU}$ is representative of $\mathrm{G}_{\mathrm{r}}$ for equatorial measurements at lower energies during this period [McDonald and Lal, 1986]. The weightedaverage value of $\mathrm{G}_{\Theta}$ for the eight solar rotations between days 136 and 343 in 1985 is $-0.36 \pm 0.05$ (or $-0.60 \pm 0.08$ ) $\% / \mathrm{deg}$ assuming $\mathrm{G}_{\mathbf{r}}=1$ (or 2) $\% / \mathrm{AU}$. This can be compared to the value of $\mathrm{G}_{\Lambda}=-0.22 \pm 0.03$ $\% /$ deg from our earlier estimate of the latitudinal com- 
ponent of the gradient obtained with a differential measurement [Christon et al., 1986] and can also be compared to the values at lower energies in the accompanying paper by McDonald and Lal [1986].

The tilt of the heliospheric current sheet is estimated from $\alpha$, the amplitude of the maximum latitudinal excursion of the heliospheric current sheet near the sun during a Carrington solar rotation (see Figure 1E), analogous to the procedure of Smith and Thomas [1986]. The maximum latitude difference is $2 \alpha$. This angular extent is taken directly from published [U.S. Dept. of Commerce, 1986] and unpublished synoptic maps of the solar magnetic field predicted on a source surface 2.5 solar radii from the sun for every solar rotation, using a potential mapping technique and Stanford solar synoptic magnetograms [Hoeksema et al., 1982,1983; Hoeksema, 1984; Smith and Thomas, 1986]. A continuous current sheet is usually well defined on this surface [Hoeksema, 1984]. The magnetic field pattern at the source surface is then assumed to be radially transported by the solar wind, controlling the configuration of the interplanetary magnetic field in the inner solar system. Distortions of the current sheet are expected at distances further from the sun due to velocity differences and stream-stream interactions (see e.g., Seuss and Hildner [1985]), but are ignored here because it is not clear that these distortions should affect the maximum latitudinal extent of the current sheet. The average value of $\alpha$ after day 121, 1985, when it reached a low, steady value, is $22.6 \pm 0.5^{\circ}$, just slightly higher than the average value of $15^{\circ}$ during the last solar minimum [Hoeksema et al., 1982].

\section{Discussion}

During 1984 and 1985 the interplanetary medium is dominated by corotating interaction regions and there is no evidence of large solar flare transient disturbances. Even if there had been some large flare initiated disturbances, a persistent inversion of flux levels as a function of radius would not be expected to be produced by large scale solar flare transients. A flux inversion might be produced by a latitude dependence of the solar wind speed or magnetic field turbulence for an extended period of time [Christon and Stone, 1985a,b; Newkirk and Fisk, 1985]. Alternatively, a negative latitudinal gradient resulting in a flux inversion may indicate that drifts are important for particle transport at this time, since the observations can be modeled using a cosmic ray transport formalism including particle drifts (see e.g., Kota and Jokipii [1983]).

In our previous paper we showed that measurements from two spacecraft, both encountering the current sheet at low to mid latitudes, will result in an average latitude gradient consistent with zero, even though a more selective analysis demonstrates that a negative latitudinal gradient is present. Average latitudinal gradients measured through 1984 are consistent with zero [Decker et al., 1984; Venkatesan et al., 1985; Webber and Lockwood, 1986]. However, in 1985, an asymmetry in the sampling of the cosmic ray flux has been introduced by the decrease of $\alpha$ to values at or lower than the latitude of Voyager 1, so that Voyager 1 now samples mainly the northern magnetic hemisphere. For example, in models including drifts, Voyager 2 should sample average equatorial fluxes which would be more intense than average fluxes at the position of Voyager 1, near the maximum latitudinal extent of the current sheet. Such a predicted inversion of tlux intensities at different radii and latitudes (applicable to this half of the 22-year magnetic-activity solar cycle) appears in Figure 3B of Kota and Jokipii [1983]. Although only qualitatively comparable because of the assumed location of the boundary of the modulation region, the model does show for $\alpha \sim 30^{\circ}$ that the equatorial flux at a given radial distance is greater than that at higher latitudes $\left(30^{\circ}\right.$ to $\left.60^{\circ}\right)$ and slightly greater distances. This apparent radial inversion of fluxes is more exaggerated for smaller values of $\alpha$.

\section{Conclusions}

The primary conclusion of this report is that the observation of $>70 \mathrm{MeV} /$ nucleon cosmic ray flux at Voyager 2 with intensities greater than those at Voyager 1 during late 1985 requires the existence of a persistent, large-scale negative latitudinal gradient. Cosmic ray flux levels at Voyager $2 \quad\left(\mathrm{r}_{2} \sim 17 \mathrm{AU}\right.$, $\left.\Theta_{2} \sim 0^{\circ}\right)$ exceed those at Voyager $1 \quad\left(r_{1} \sim 24\right.$ AU, $\left.\Theta_{1} \sim 26^{\circ} \mathrm{N}\right)$ after the current sheet tilt decreases to values close to those observed during the last solar minimum. The appearance of the persistent negative latitudinal gradient at the Voyagers occurs during a period of relatively low solar activity and is not associated with the passage of transient solar disturbances. Its appearance may, however, be associated with the large decrease in the latitudinal amplitude of the heliospheric current sheet suggested by observations of the solar magnetic field.

Acknowledgements. We are grateful to R.E. Vogt for his contributions to the Cosmic Ray Subsystem on the Voyager spacecraft. We appreciate the contributions of the other Voyager Cosmic Ray Subsystem team members consisting of scientists and engineers at the California Institute of Technology, the Goddard Space Flight Center, the University of Arizona, and the University of New Hampshire. Work at Caltech was supported in part by the National Aeronautics and Space Administration under contract JPL 49-55663120-0-2600 and under grant NGR 05-002-160. Work at Stanford was supported in part by the National Aeronautics and Space Administration under Grant NGR5-020-559, the Office of Naval Research under contract N00014-76-C-0207, and the Atmospheric Sciences Section of the National Science Foundation under Grant ATM77-20580.

\section{References}

Burlaga, L. F., F. B. McDonald, M. L. Goldstein, and A. J. Lazarus, Cosmic ray modulation and turbulent interaction regions near $11 \mathrm{AU}, \underline{\mathrm{J}}$. Geophys. Res., 90, $12027,1985$. 
Christon, S. P., A. C. Cummings, E. C. Stone, K. W. Behannon, L. F. Burlaga, J. R. Jokipii, and J. Kota, Differential measurement and model calculations of cosmic ray latitudinal gradient with respect to the heliospheric current sheet, J. Geophys. Res., 91, 2867, 1986.

Christon, S. P. and E. C. Stone, Latitude variation of recurrent $\mathrm{MeV}$-energy proton fluxes enhancements in the heliocentric radial range 11 to $20 \mathrm{AU}$ and possible correlation with solar coronal hole dynamics, Geophys. Res. Lett., 12, 109, 1985 a.

Christon, S. P. and E. C. Stone, Recurrent MeV-energy proton fluxes beyond $15 \mathrm{AU}$ : latitude variations and possible correlation with coronal hole dynamics, EOS Trans. Am. Geophys. U., 66, 1013, 1985 b.

Decker, R. B., S. M. Krimigis, and D. Venkatesan, Estimate of cosmic-ray latitudinal gradient in 1981-1982, Astrophys, J. Lett., 278, L119, 1984.

Hoeksema, J. T., J. M. Wilcox, and P. H. Scherrer, Structure of the heliospheric current sheet in the early portion of sunspot cycle 21 , J. Geophys. Res. 87. $10331,1982$.

Hoeksema, J. T., J. M. Wilcox, and P. H. Scherrer, The structure of the heliospheric current sheet: 1978-1982, J. Geophys. Res., 88, 9910, 1983.

Hoeksema, J. T., Structure and evolution of the large scale heliospheric magnetic fields, Ph.D. Thesis, Stanford, University, Stanford, CA, 1984.

Kota, J. and J. R. Jokipii, Effects of drift on the transport of cosmic rays. VI. A three dimensional model including diffusion, Astrophys. J.. 265, 573, 1983.

McDonald, F. B., and N. Lal, Variations of galactic cosmic rays with heliolatitude in the outer heliosphere, Geophys. Res. Lett. this issue, 1986.

Newkirk, G., Jr., and L. A. Fisk, Variation of cosmic rays and solar wind properties with respect to the heliospheric current sheet 1 . Five $\mathrm{GeV}$ protons and solar wind speed, J. Geophys. Res., 90, 3391, 1986.
Roelof, E. C., R. B. Decker, and S. M. Krimigis, Latitudinal and field aligned cosmic ray gradients 2 to 5 AU Voyagers 1 and 2 and IMP 8, J. Geophys. Res., 88, 9889, 1983.

Seuss, S. T. and E. Hildner, Deformation of the heliospheric current sheet, J. Geophys. Res., 90, 9461, 1985.

Smith, E. J. and B. T. Thomas, Latitudinal extent of the heliospheric current sheet and modulation of galactic cosmic rays, J. Geophys. Res., 91, 2933, 1986.

Stone, E. C., R. E. Vogt, F. B. McDonald, B. J. Teegarden, J. H. Trainor, J. R. Jokipii, and W. R. Webber, Cosmic ray investigation for the Voyager missions; energetic particle studies in the outer heliosphere and beyond, Space Sci. Rey.. 21, 335, 1977.

U. S. Department of Commerce, The solar magnetic field -- 1976 through 1985, Report UAG-94, NOAA, Boulder, CO., 1986.

Venkatesan, D., R. B. Decker, and S. M. Krimigis, Voyager 1 and 2 measurements of radial and latitudinal cosmic ray gradients during 1981-84, Proc. Int. Cosmic Ray Conf. 19th, 7, 202, 1985.

Webber, W. R. and J. A. Lockwood, Interplanetary cosmic-ray radial and latitudinal gradients derived in 1984 using IMP 8, Voyager, and Pioneer data, Astrophys. J. 302, 511, 1986.

S. P. Christon, Johns Hopkins University Applied Physics Laboratory, Laurel, MD 20707.

E. C. Stone, 103-33, California Institute of Technology, Pasadena, CA 91125.

J. T. Hoeksema, Institute for Plasma Research, Stanford University, Stanford, CA 94305

(Received June 2, 1986; accepted June 23, 1986) 